\title{
PARALLEL DETERMINATION OF DESOGESTREL AND 17 $\alpha$-ETHINYLESTRADIOL IN PHARMACEUTICAL FORMULATION BY DERIVATIVE SPECTROPHOTOMETRY
}

\author{
M. INÉS TORAL*, FALLON NACARATTE, FRANCISCA NOVA-RAMÍREZ, ROMINA OTIPKA
}

Department of Chemistry, Faculty of Sciences, University of Chile, PO Box 653, Santiago, Chile.

(Received: January 18, 2013 - Accepted: March 12, 2013)

\begin{abstract}
This work presents a rapid and simple method for the parallel determination of Desogestrel (DSG) and 17 $\alpha$-Ethinylestradiol (EE2) by second order derivative spectrophotometry and its application in pharmaceutical formulations. Study of the effect of the solvents, excipients and spectral behavior is also included. Acetonitrile was selected as a solvent; subsequently the samples were evaluated by second order derivatives, using a smoothing factor of 8,000 and a scale factor of $10^{4}$. The EE2 determination was carried out using the graphical method at $288 \mathrm{~nm}$ and DSG by the zero-crossing method at $220 \mathrm{~nm}$. The determination ranges were found to be between $1.5 \cdot 10^{-6}$ and $5 \cdot 10^{-4} \mathrm{~mol} / \mathrm{L}$ and $1.7 \cdot 10^{-7}$ to $1.0 \cdot 10^{-3} \mathrm{~mol} / \mathrm{L}$ for DSG and EE2, respectively.

The homogenized tablets are divided in two fractions, the first, called sample A, was dissolved in acetonitrile, which contains the absorbents species that is polyvidone, DGS and EE2, the latter is determined directly at $288 \mathrm{~nm}$. Meanwhile, in the second fraction (sample B), after extract with water the polyvidone and partially EE2, it is possible determine DGS at $220 \mathrm{~nm}$. When the pharmaceutical formulation contains Vitamin E (Vit. E) is necessary to use an equations system to be evaluated in sample B.
\end{abstract}

Key words: Spectral study; Desogestrel; 17 $\alpha$ - Ethinylestradiol; Povidone; Pharmaceutical formulations.

\section{INTRODUCTION}

The female contraceptive methods found in the pharmaceutical market have a wide range of presentations. However, the barrier and oral hormonal types have a greater efficiency and effectiveness in terms of family planning. The latter are the estrogen and/or progesterone type prescribed individually or in combination; for example, $17 \alpha$-Ethinylestradiol and Desogestrel.

The 17 $\alpha$-Ethinylestradiol (EE2) corresponds to 17 $\alpha$-Ethynyl-1,3,5(10)estratriene-3,17 $\beta$-diol,19-Nor-1,3,5(10),17 $\alpha$-pregna-trien-20-yne-3,17-diol (Figure 1a) is the first semi-synthetic estrogen to be developed synthetized from estradiol (E2), this drug is useful by the oral route, because the ethinyl group added in the 17th position gives it greater resistance to degradation in the human body. The mechanism of action consists of different exchange rates. In the hypothalamus the release of luteinizing hormone (LH) and follicle stimulating hormone (FSH) slows down, which also means decreased rates of ovulation and folliculogenesis. This compound has MW $294.4 \mathrm{~g} / \mathrm{mol}$; the solubility in water is between 4.8 to $11.3 \mathrm{mg} / \mathrm{L}$; $\log$ Kow $3.6^{1}$ and their $\mathrm{pKa}$ is $10.4^{2-3}$.
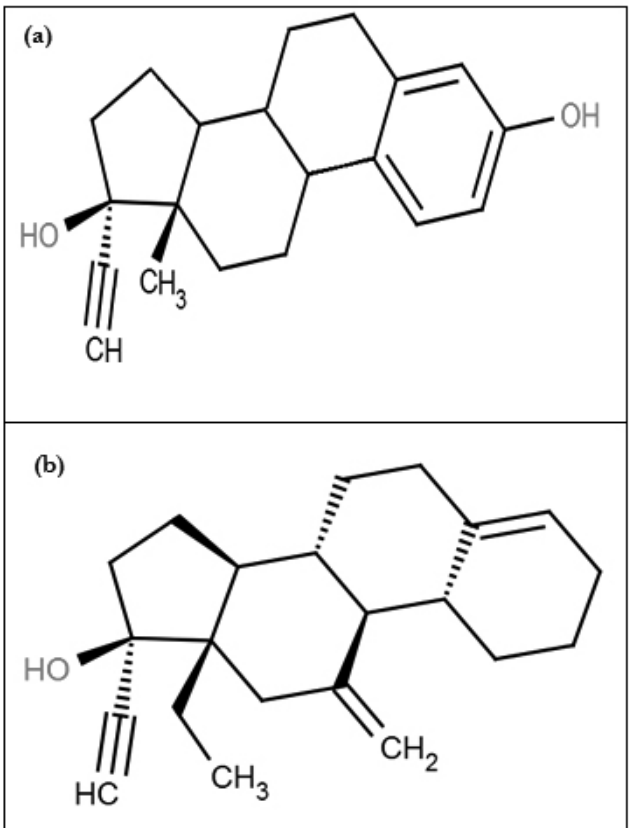

Figure 1. Structures of the analytes (a) Ethinylestradiol (EE2) and (b) Desogestrel (DSG).
Desogestrel (DSG), is 13-Ethyl-11-methylene-18,19-dinor-17 $\alpha$-4pregnen-20-yn-17-ol), (Figure 1b), which has MW of $310.6 \mathrm{~g} / \mathrm{mol}$; the solubility in water is $0.5 \mathrm{mg} / \mathrm{L}$; $\log$ Kow $5.6^{4}$ and their $\mathrm{pKa}$ is 10.4 . This drug is an active progestin which is metabolized to the active form Etonogestrel ${ }^{5}$. The mode of action is to simulate ovulation; it produces a morphological transformation in the endometrium.

The combination of EE2 and DSG (known as third generation birth control pills) produces a synergistic effect in their mechanisms of action on the body, producing a high potency effect that suppresses the level of activity of the hypothalamus and pituitary gland.

Analytical methods using different techniques have been reported for the determination of EE2 together with other drugs; for example, UV-Vis derivative spectrophotometry in different orders of derivative $e^{6-7-8}$, as well as the chromatographic type such as $\mathrm{GLC}^{9}, \mathrm{GC}^{10}, \mathrm{HPLC}^{11-12}$ and HPLC/ESIMS/ $\mathrm{MS}^{13}$. The determination of DSG has been reported together with other drugs including EE2, using different techniques; for example, HPLC ${ }^{14-15}$, HPLC/MS ${ }^{16}$, HPLC/MS/MS ${ }^{17-18}$. Published methods for the simultaneous determination of both compounds require careful preparation of the column, detector, and also expensive equipment. For this reason, it is necessary to develop a simple, rapid, inexpensive, and sensitive method.

This paper describes the development of the determination of DSG and EE2 through an alternative method that is economical, rapid and sensitive by second derivative spectrophotometry. For this, it was necessary to select the best solvent for the analysis, to optimize the chemical and spectral variables and apply the proposed method to the determination of $17 \alpha$-Ethinylestradiol and Desogestrel in tablets.

\section{MATERIAL AND METHODS}

\section{Apparatus}

A Shimadzu (Shimadzu Co., Kyoto, Japan) UV-1603PC spectrophotometer with $10 \mathrm{~mm}$ quartz cells was used to measure the absorbance and derivative spectra. The baseline was corrected for a set of experiments and the spectra was recorded over a range of 190 to $350 \mathrm{~nm}$ against the corresponding solvent. The data were obtained by software Shimadzu, kit version 3.7 (P / N 206-60570 04). A Sartorius R 200D balance with an uncertainty of $\pm 0.01 \mathrm{mg}$, a Simplicity Millipore deionizer 185, a vortex mixer KMC-1300V model and an Eppendorf centrifuge 5430 were also used.

\section{Reagents and Solvents}

All reagents were of analytical quality. EE2 (98\% purity) and DSG $(99.7 \%$ purity) were supplied by Sigma-Aldrich. Stock solutions of $2.0 \cdot 10^{-4} \mathrm{~mol} / \mathrm{L}$ were prepared by dissolving $1.55 \pm 0.01 \mathrm{mg}$ and $1.48 \pm 0.01 \mathrm{mg}$ for DSG and EE2, respectively, in $25 \mathrm{~mL}$ of acetonitrile. Similarly, for the study of the solvent effect on the spectral behavior of DSG and EE2, the same process was conducted using methanol, ethanol, hexane, ethyl acetate, dichloromethane, distilled water and chloroform.

Then appropriate dilutions were prepared in concentrations ranges of 
$1.0 \cdot 10^{-5}$ to $10 \cdot 10^{-5} \mathrm{~mol} / \mathrm{L}$ for each solvent. The pharmaceutical formulations Ciclidon ${ }^{\circledR}(0.150 \mathrm{mg}$ DSG and $0.030 \mathrm{mg}$ EE2 $)$ and Dal ${ }^{\circledR}(0.150 \mathrm{mg}$ DSG and $0.020 \mathrm{mg}$ EE2) were obtained from the Recalcine S.A. Laboratory. The interference study was performed with different excipients (microcrystalline cellulose, polyvidone, talc, magnesium stearate, aluminum lake, lactose, starch, macrogol and hydroxypropylcellulose). Stock solutions of $1.0 \cdot 10^{-3} \mathrm{~mol} / \mathrm{L}$ were prepared in acetonitrile, then were diluted and measured. Vacuum filtration with PVDF filter paper was used for this method.

\section{Procedures}

\section{Calibration of EE2 and DSG}

Different aliquots of the stock solution of EE2 were individually diluted in $5 \mathrm{~mL}$ of acetonitrile in the concentration range of $1.0 \cdot 10^{-5}$ to $9.0 \cdot 10^{-5} \mathrm{~mol} / \mathrm{L}$ and then was homogenized for $1 \mathrm{~min}$. The same procedure was performed for DSG. In all cases, the corresponding absolute values of the second order derivative spectra at $220 \mathrm{~nm}$ and $288 \mathrm{~nm}$ for DSG and EE2 were obtained and the values of derivative unit (DU) versus concentration were plotted.

Procedure for the simultaneous determination of EE2 and DSG in synthetic mixture with different mass relations

Different aliquots of stock solutions of EE2 and DSG were simultaneously diluted in acetonitrile over a concentration range of $2.0 \cdot 10^{-5}$ to $6.0 \cdot 10^{-5} \mathrm{~mol} / \mathrm{L}$ of DSG in the presence of $1.0 \cdot 10^{-5} \mathrm{~mol} / \mathrm{L}$ of EE2. The same procedure was performed for EE2 in a concentration range of $1.0 \cdot 10^{-5}$ to $4.0 \cdot 10^{-5} \mathrm{~mol} / \mathrm{L}$ in the presence of $5.0 \cdot 10^{-5} \mathrm{~mol} / \mathrm{L}$ of DSG. Each solution was performed with five repetitions and then the respective wavelengths were evaluated.

Study of Stress testing of forced degradation

For photo-stability studies, solutions of $1.0 \cdot 10^{-5} \mathrm{~mol} / \mathrm{L}$ of EE2 and $5.0 \cdot 10^{-5}$ $\mathrm{mol} / \mathrm{L}$ of DSG were prepared using acetonitrile. The individual solutions were exposed to direct light, indirect light and darkness between $1 \mathrm{~h}$ and $7 \mathrm{~h}$, and then the second order derivative spectra were evaluated each $1 \mathrm{~h}$ for all solutions. Similarly, the study was performed under reductive $(\mathrm{Zn} 0.1 \mathrm{~mol} / \mathrm{L})$, oxidative $\left(\mathrm{H}_{2} \mathrm{O}_{2} 0.1 \%\right)$, and acid-base $(\mathrm{HCl}$ and $\mathrm{NaOH}$ both at $0.1 \mathrm{~mol} / \mathrm{L})$ conditions, and the different solutions were evaluated after $1 \mathrm{~h}$ of constant agitation.

Procedure for determination of EE2 and DSG in pharmaceutical formulations

The content of 10 tablets of Ciclidon ${ }^{\circledR}$ were weighted and powdered during 10 min and the sample was divided into 2 samples of equal mass. Sample A was used to determinate EE2; this was diluted in $25 \mathrm{~mL}$ of acetonitrile, and then was shaken for $5 \mathrm{~min}$ and centrifuged for $5 \mathrm{~min}$ at $5000 \mathrm{rpm}$. DSG was determined in sample B. This was diluted in $30 \mathrm{~mL}$ of deionized water, shaken for $10 \mathrm{~min}$, then was filtered, the supernatant was discarded and finally the solid was diluted in $25 \mathrm{~mL}$ acetonitrile and was shaken for $5 \mathrm{~min}$ and filtered once more. The same procedure was used for $\mathrm{Dal}{ }^{\circledR}$, but sample $\mathrm{A}$ was diluted to $12.5 \mathrm{~mL}$. According to the nominal content, the stock concentrations for Ciclidon ${ }^{\circledR}$ and Dal ${ }^{\circledR}$ were $2.02 \cdot 10^{-5} \mathrm{~mol} / \mathrm{L}$ and $2.70 \cdot 10^{-5} \mathrm{~mol} / \mathrm{L}$, respectively to $\mathrm{EE} 2$; and $9.66 \cdot 10^{-5} \mathrm{~mol} / \mathrm{L}$ to DSG in both formulations. Then, $2.5 \mathrm{~mL}$ from the supernatant of the samples individually (A and B) was diluted with acetonitrile to $5 \mathrm{~mL}$ and then was evaluated by second derivative spectrophotometry. In the case of Dal, $2 \mathrm{~mL}$ was diluted from the supernatant.

\section{RESULTS AND DISCUSSION}

\section{Study preliminaries and Optimization of chemical variables}

Among the excipients of the pharmaceutical formulations containing DSG and EE2 are found polyvidone (PVP) and in some cases vitamin E (Vit.E). This makes it difficult the simultaneous determination by spectrophotometry of these contraceptives, because the PVP and Vit.E, absorb in the same region as DSG, as can be seen in Figure 2.

Given this situation, we conducted a study of solubilities and EE2 spectral behavior, DSG and PVP in different solvents, being that DSG and EE2 are soluble in various organic solvents among them acetonitrile, in which its bands are well defined. However, $\mathrm{PVP}^{19}$ present a favorable solubility in this solvent (Figure 3).

The Vit.E is soluble in organic solvents as well as DSG and EE2, which make impossible its separation. The proposed method can be applied to those pharmaceuticals containing this compound using an equation system.

With respect to PVP its solubility was studied in different solvents to avoid its possible interference in this analysis through its extraction or their insolubility, for this reason was dissolved in different solvents like hexane, ethyl acetate, distilled water, acetone, acetic acid $0.2 \mathrm{~mol} / \mathrm{L}$ and methanol. In the first two, PVP presented a partial solubility, whereas in the others, the solubility was total. However experimentally, the observation was that every solution presented great signals of absorbance in a range of $\lambda 190$ a $250 \mathrm{~nm}$, so this polymer, known as having universal solubility ${ }^{20-21}$, presented interference in every case.

Although PVP it is soluble in any type of solvent, the analytes are not soluble, for this reason it was considered one previous solubilization process in water, which could be a way to eliminate the PVP interference, however the EE2 also presented a partial solubility in this solvent.
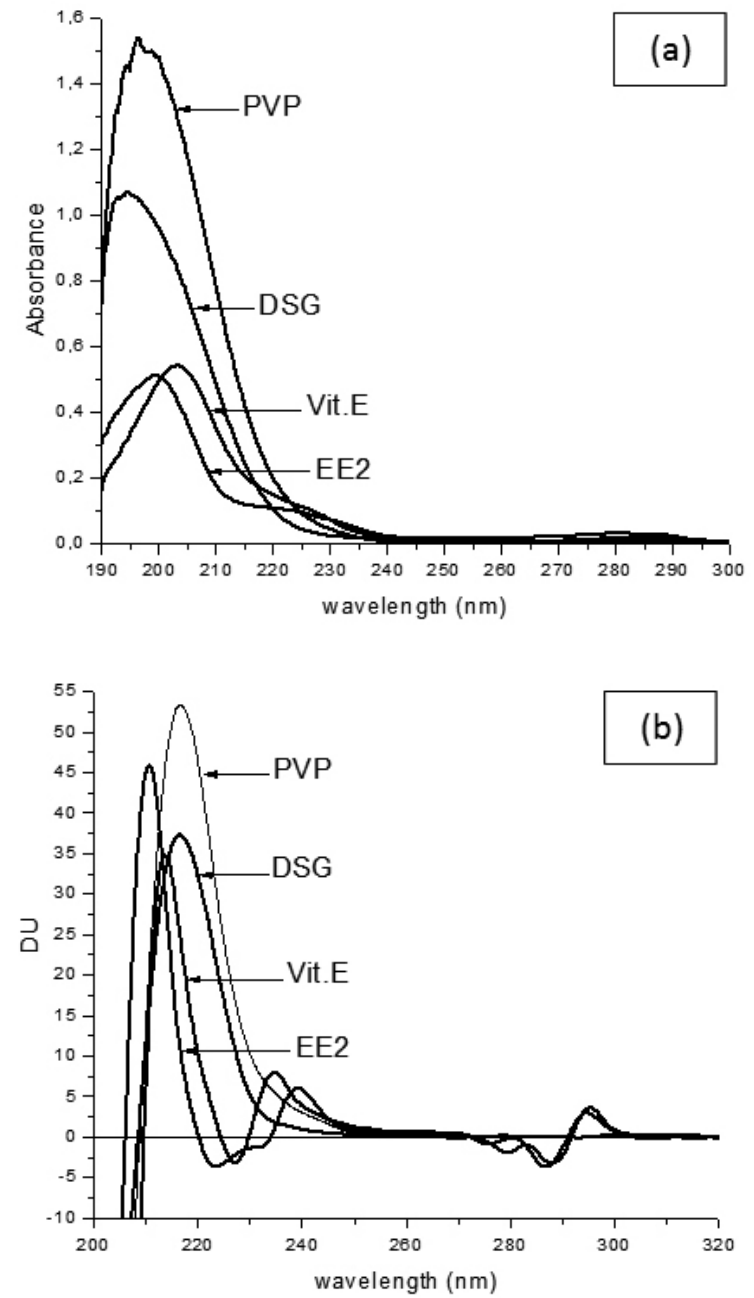

Figure 2. (a) Clasical spectra of DSG, EE2, Vit. E and PVP, (b) Second order derivative spectra of DSG, EE2, Vit. E and PVD. The concentrations of EE2 and Vit.E are $1 * 10^{-5}(\mathrm{~mol} / \mathrm{L})$, DSG is $5^{*} 10^{-5}(\mathrm{~mol} / \mathrm{L})$ and PVP is $1 * 10^{-6}$ $(\mathrm{mol} / \mathrm{L})$.

In this context, it was decided a parallel extraction of DSG and EE2 and subsequent determination in acetonitrile using derivative spectrophotometry, the developed method will allow application in pharmaceutical formulations containing such excipient,

Despite this method uses previous stages, represents a significant contribution since utilizes low cost instrumentation.

Therefore, considering these aspects were optimized the spectral variables for each analyte, considering the best conditions to favor the parallel optimization of the analytes.

In all cases each drug was evaluated in $\mathrm{AN}$, therefore the determination was optimized in this solvent using the derivative technique, to avoid interference of small amounts of incomplete extraction. 

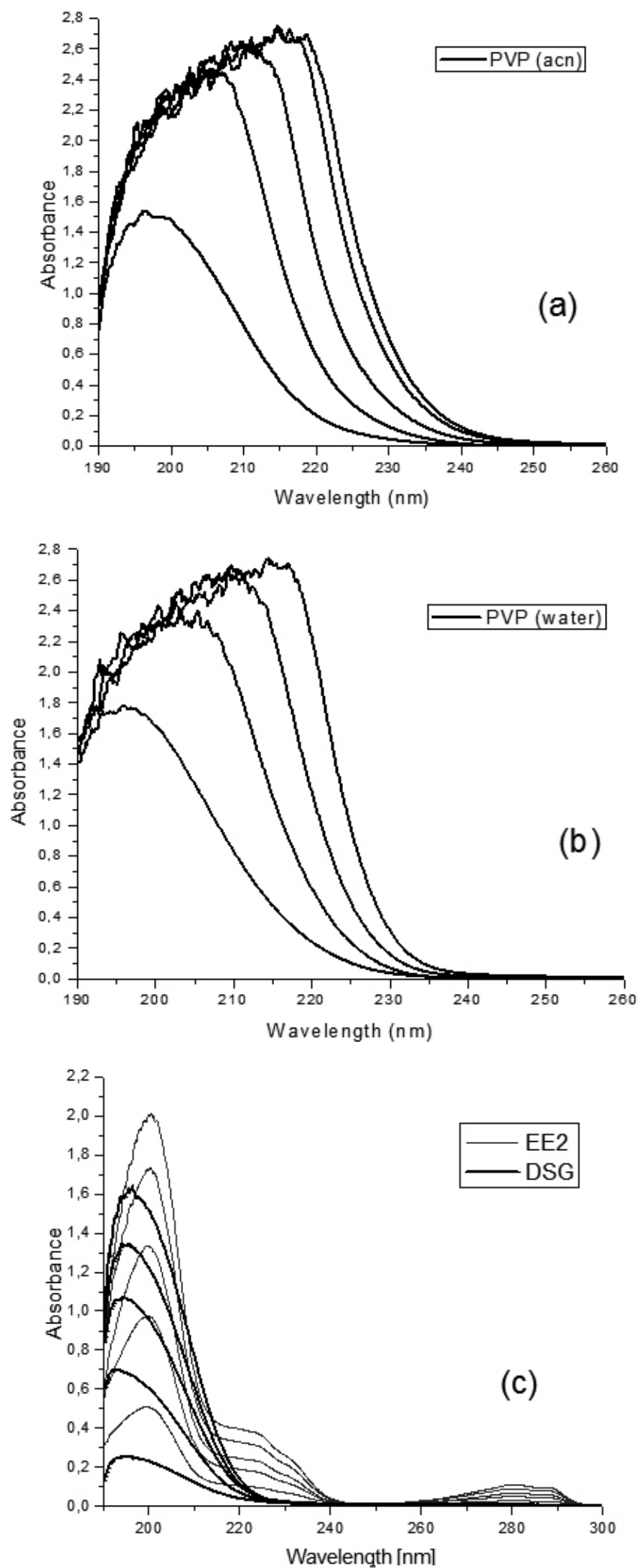

Figure 3. Classical spectra of the excipients. (a) PVP dissolved in acetonitrile in a concentration range from $1 * 10^{-6}$ to $12 * 10^{-6}(\mathrm{~mol} / \mathrm{L})$, (b) PVP dissolved in water in a concentration range of $1 * 10^{-6}$ to $9 * 10^{-6}(\mathrm{~mol} /$ L) and (c) DSG and EE2 dissolved in acetonitrile in a concentration range of $1.0 \cdot 10^{-5}$ to $5.0 \cdot 10^{-5} \mathrm{~mol} / \mathrm{L}$ and $1.0 \cdot 10^{-5}$ to $9.0 \cdot 10^{-5} \mathrm{~mol} / \mathrm{L}$ respectively.
Study of Stress testing of forced degradation

The forced stability study was made in agreement with procedure. In photo-stability with direct and indirect light and darkness conditions no photochemical degradation was observed in the spectral bands of either analyte. Neither was any change observed in the presence of reductive conditions like $\mathrm{Zn}$. In the study of oxidative stress for EE2 and DSG, only noise was observed.

In conditions of acid hydrolysis, in the case of EE2, a bathochromic shift of $\Delta \lambda=3.8 \mathrm{~nm}$ was observed in the primary band $(\lambda 190$ to $212 \mathrm{~mm}$ ) and a hypsochromic effect of $\Delta \lambda$ max $=2.4 \mathrm{~nm}$ was observed in the secondary band (300 to $270 \mathrm{~nm}$ ). This effect can be attributed to hydrogen bridge formations, avowing to the polarity of the solvent. In the case of DSG, a bathochromic effect of $\Delta \lambda_{\max }=8.1 \mathrm{~nm}$ was observed, because solvent polarity stabilizes the excited state of the functional groups of the analyte. In basic hydrolysis of $\mathrm{pH}>12, \mathrm{EE} 2\left(\mathrm{pKa}=10.40^{(17-18)} \mathrm{pH}>\mathrm{pKa}\right.$, the analyte is in its deprotonated form. In the case of DSG, a bathochromic and hypochromic shift was also observed. These effects ensued because of the stabilization of the excited state, since this solvent has a great polarity. In this context, it is necessary to avoid the oxidative and acid-base conditions and work only with the acetonitrile solvent because it presents a better stability and high sensitivity of the analytes.

Polividone was subjected to the same stress situation, finding no effect on its spectra.

Selection of derivative order

To select the derivative order, the derivatives of first to fourth order were obtained (Figure 4) using a range of $\lambda$ between 190 to $350 \mathrm{~nm}$, a smoothing factor of 8,000 and a scaling factor 104. In the first order derivative (Figure 4a), zones characteristic for this simultaneous determination were not present, so this order was discarded. In the second order derivative (Figure 4b), the signals decrease, but the spectra were well-defined and present linearity between absorbance and concentration for both analytes. The upper level derivative orders were discarded (Figure $4 \mathrm{c}$ and $4 \mathrm{~d}$ ) because they presented a low signal/ noise ratio and a decreasing sensitivity.
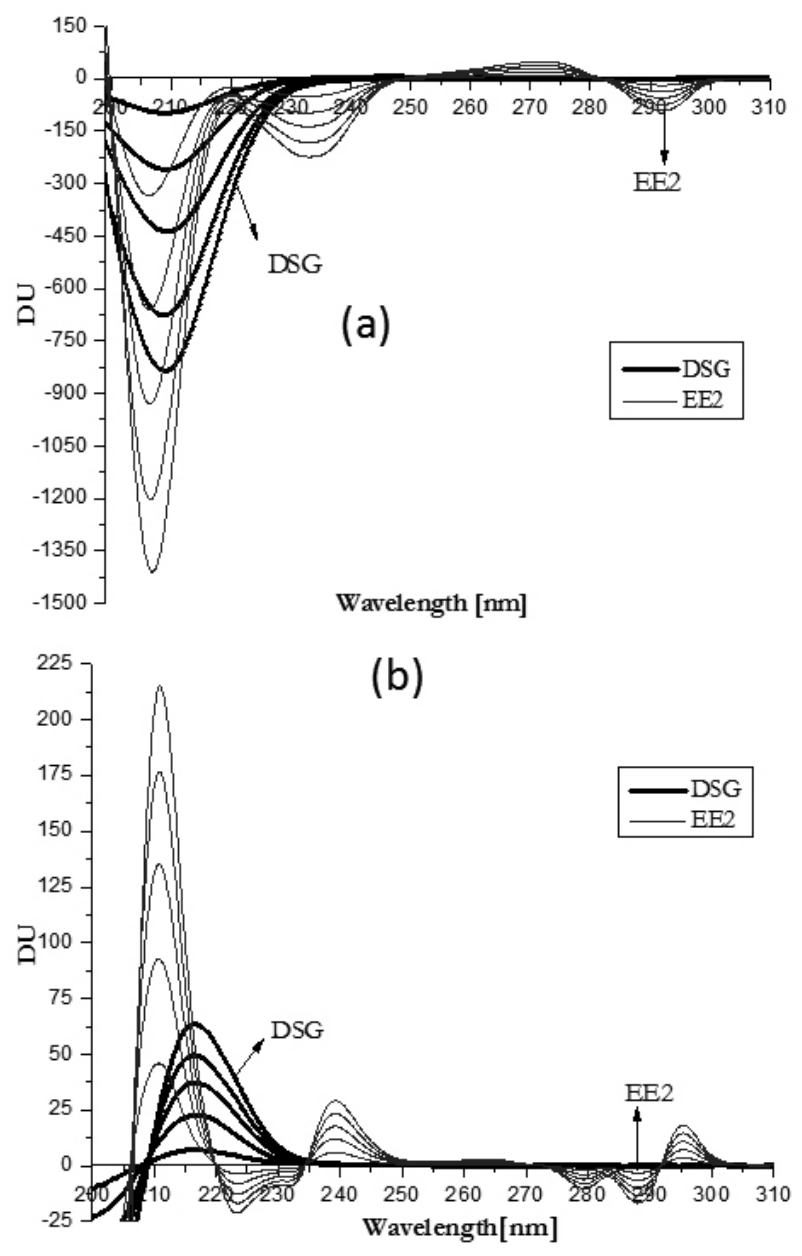

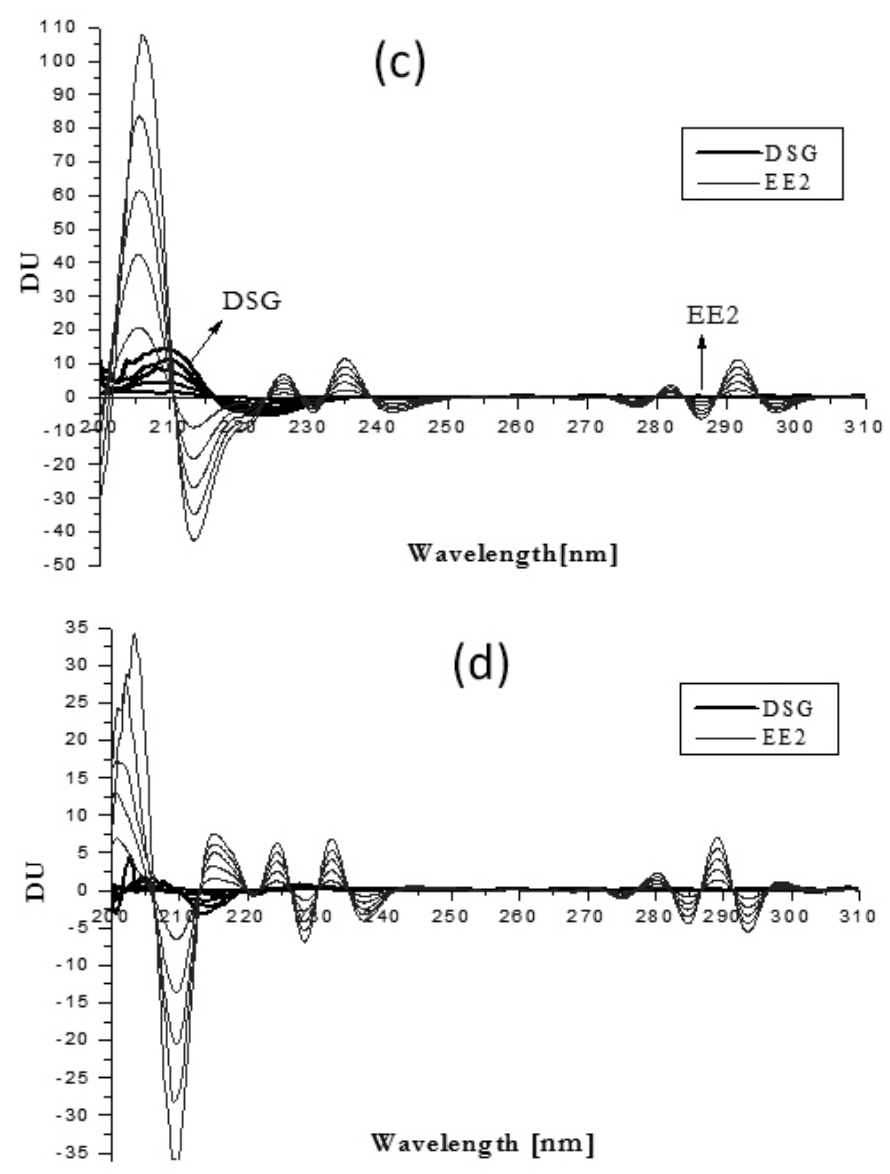

Figure 4. Derivative spectra of (a) first, (b) second, (c) third and (d) fourth order for EE2 and DSG in a concentration range from $1.0 \cdot 10^{-5}$ to $5.0 \cdot 10^{-5} \mathrm{~mol} / \mathrm{L}$ and $1.0 \cdot 10^{-5}$ to $9.0 \cdot 10^{-5} \mathrm{~mol} / \mathrm{L}$, respectively.

Is necessary to highlight that the polyvidone not absorb between 280 and $310 \mathrm{~nm}$ (Figure 2), for this reason EE2 can be determined in its presence.

Selection of Smoothing Factor and Scale Factor

The second derivative was used as the optimization smoothing factor given by the program,

using 1000, 2000, 4000 and 8000 values (a scale factor of was $10^{4}$ considered as a constant value). The Figure 5 shows that when the smoothing value increases, the noise of the signal decreases but with a loss of sensitivity. However, taking into consideration the definition of the signal, the factor of 8000 was chosen (Figure 5d) as the optimum value, because both signals DSG and EE2 were well-defined.

A scaling factor of $10^{4}$ was selected in order to obtain a good reading of the spectral bands of both analytes, because this factor does not alter the detection and quantification limits.

Selection of analytical wavelength

Using the parameters of the second derivative, a smoothing factor of 8,000 and a scaling factor of $10^{4}$, we proceeded to the selection of the analytical wavelength for both analytes. In derivative spectra, it was observed that EE2 could be determined by the graphical method in a wavelength range between 270 to $300 \mathrm{~nm}$. Because DSG does not present a signal in this range, the wavelength chosen was $288 \mathrm{~nm}$, since the signal is higher, which increases the sensitivity of the method. To determine DSG, zerocrossing presented a welldefined wavelength to $220 \mathrm{~nm}$, while the derivative spectra of DSG increased with the concentration of this drug. Therefore, this wavelength was chosen.

\section{Analytical Parameters}

In agreement with the procedure the calibration curve was done with nine points for DSG and EE2, a good linearity by linear regression for both analytes was obtained (Table 1). Besides, eleven independent blanks were used to determinate LD and LC for DSG and EE2 where the $\sigma$ was the standard deviation of blank. Accuracy was determined according to the \% recovery and the reproducibility expressed as relative standard deviation (RSD), which were obtained with nine individual solutions containing the pharmaceutical proportion $1: 5,1.0 \cdot 10^{-5} \mathrm{~mol} / \mathrm{L}$ for EE2 and $5.0 \cdot 10^{-5} \mathrm{~mol} / \mathrm{L}$ for DSG on different days. A recovery was obtained between $97.7 \%-101.0 \%$ for EE2 and between $97.2 \%-102.0 \%$ for DSG, obtaining RSD less than to $2 \%$.

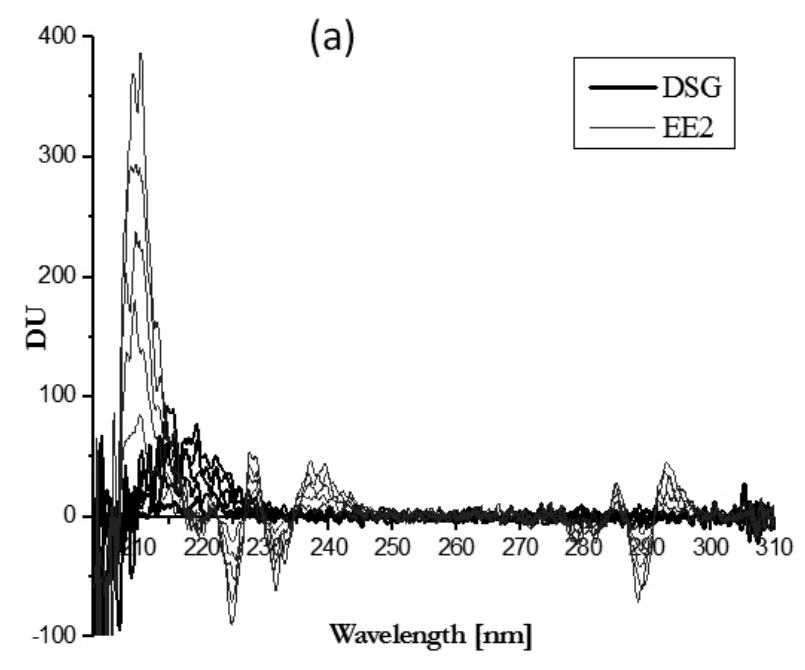

(b)
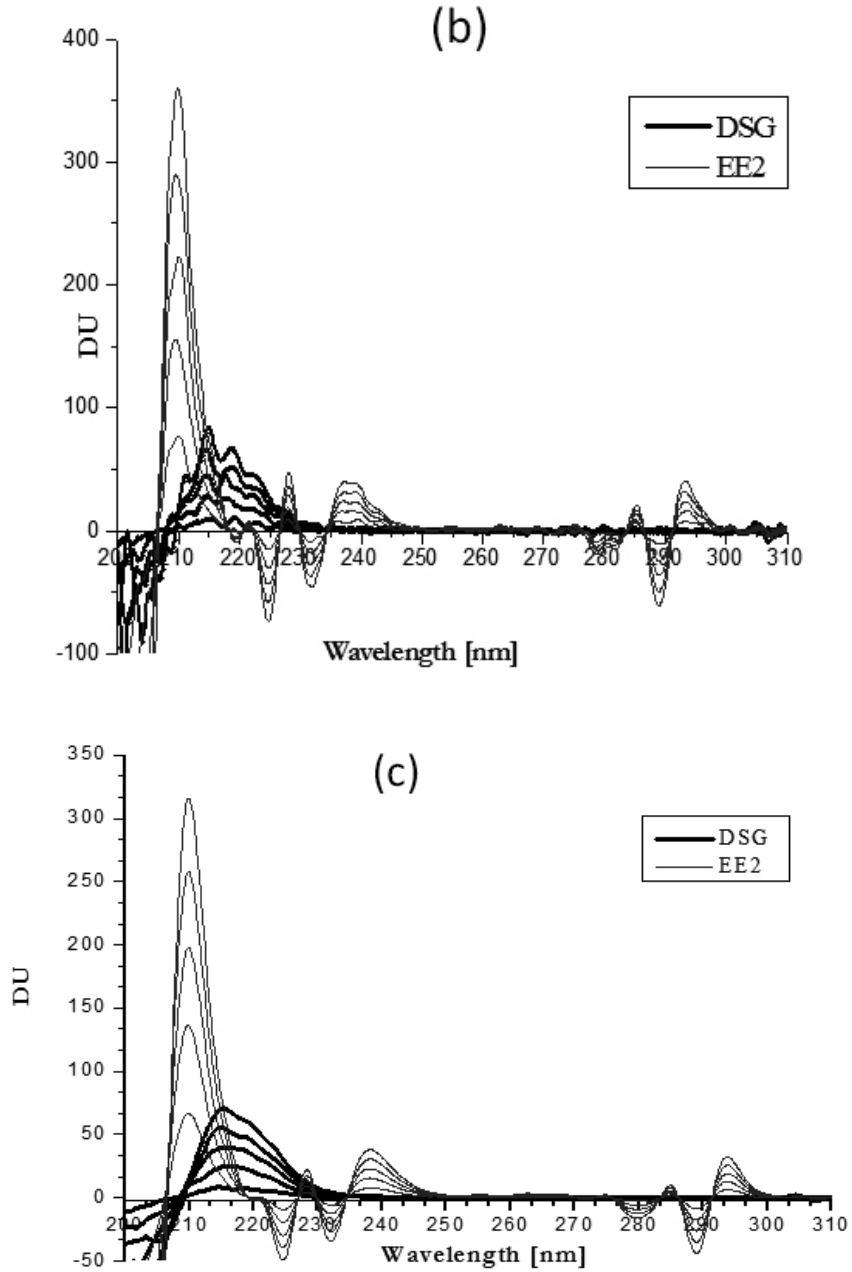


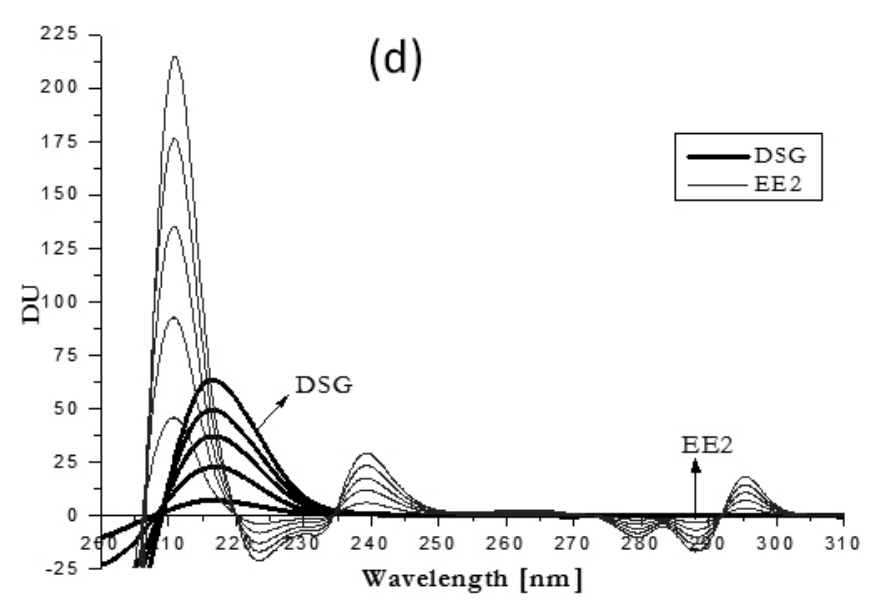

Figure 5. Derivative spectra of second order with different smoothing of EE2 and DSG. Smoothing factor (a) $1 \cdot 10^{3}$, (b) $2 \cdot 10^{3}$, (c) $4 \cdot 10^{3}$ and (d) $8 \cdot 10^{3}$. Concentration range $1.0 \cdot 10^{-5}-5.0 \cdot 10^{-5} \mathrm{~mol} / \mathrm{L}$ and $1.0 \cdot 10^{-5}-9.0 \cdot 10^{-5} \mathrm{~mol} / \mathrm{L}$ for EE2 and DSG, respectively.

Simultaneous determination of EE2 and DSG for different ratios in mass on the wavelength.

As was mentioned, the method was oriented to a parallel determination because of the PVP interference in DGS determination because absorbs in the same region.

However, each analyte can be partially dissolved in the extraction of the other, and the quantification of each drug must be made in the presence of a small amount of other drug. In this context, the derivative technique was used, so that the study of different ratios of mass was established, where one of the analytes has a constant mass and other has a variable mass, determining EE2 and DSG simultaneously without the presence of PVP.

In Figure $6 \mathrm{a}$ and $6 \mathrm{~b}$, the wavelengths were observed to be well-defined in the area of constant concentration of the analyte of the sample. Then, each ratio was determined by the percent of recuperation for both analytes (Table 2), in which good recoveries were observed for DSG and EE2, which were between $95 \%$ to $105 \%$.
Table 1. Parameters Analytical.

\begin{tabular}{|c|c|c|}
\hline Parameters & DSG & EE2 \\
\hline Analytical wavelength [nm] & 220 & 288 \\
\hline Slope & $6.17 \cdot 10^{5}$ & $3.33 \cdot 10^{5}$ \\
\hline Intercept & $-0,82$ & $-4.29 \cdot 10^{-2}$ \\
\hline Correlation coefficient & 0.9991 & 0.9999 \\
\hline $\begin{array}{c}\text { Limit of detection (LOD)* } \\
\text { (mol/L) }\end{array}$ & $4.9 \cdot 10^{-7}$ & $5.5 \cdot 10^{-8}$ \\
\hline $\begin{array}{c}\text { Limit of quantification } \\
\text { (LOQ)** (mol/L) }\end{array}$ & $1.5 \cdot 10^{-6}$ & $1.7 \cdot 10^{-7}$ \\
\hline $\begin{array}{c}\text { Determination range RD } \\
\text { (mol/L) }\end{array}$ & $1.5 \cdot 10^{-6}-5.0 \cdot 10^{-4}$ & $1.7 \cdot 10^{-7}-1.0 \cdot 10^{-3}$ \\
\hline Reproducibility (RSD, \%) & 1.62 & 1.43 \\
\hline *LOD: 3.3s, **LOQ: 10s. & \multicolumn{2}{|l}{} \\
\hline
\end{tabular}

Table 2. Simultaneous determination of EE2 and DSG in different ratios of mass.

\begin{tabular}{|c|c|c|c|c|}
\hline \multirow{2}{*}{$\begin{array}{l}\text { DSG: } \\
\text { EE2 }\end{array}$} & \multicolumn{2}{|c|}{ Added mass (mg) } & \multicolumn{2}{|c|}{$\begin{array}{l}\text { Mass obtained (mg) y } \\
\text { recovery }(\%)^{*}\end{array}$} \\
\hline & DSG $\cdot 10^{-2}$ & EE2 $\cdot 10^{-2}$ & DSG $\cdot 10^{-2}$ & EE2 $\cdot 10^{-2}$ \\
\hline $2: 1$ & 6.2 & 2.96 & $6.30(101.4)$ & $3.00(101.1)$ \\
\hline $3: 1$ & 9.3 & 2.96 & $9.30(99.8)$ & $2.99(100.8)$ \\
\hline $4: 1$ & 12.4 & 2.96 & $12.5(100.6)$ & $2.96(99.7)$ \\
\hline $5: 1$ & 15.5 & 2.96 & $15.3(98.3)$ & $3.03(102.1)$ \\
\hline $6: 1$ & 18.6 & 2.96 & $18.5(99.1)$ & $2.95(99.6)$ \\
\hline $5: 2$ & 15.5 & 5.93 & $15.3(98.6)$ & $5.98(100.9)$ \\
\hline $5: 3$ & 15.5 & 8.89 & $14.9(96.2)$ & $9.02(101.5)$ \\
\hline $5: 4$ & 15.5 & 11.9 & $14.4(95.0)$ & $12.1(102.1)$ \\
\hline
\end{tabular}

Parallel determination of EE2 y DGE in pharmaceutical formulation

The parallel determination of EE2 y DGE in pharmaceutical formulation was carried out with six replicas, according to procedure and Schema I.

Contents obtained equivalent in mass to the formulation pharmaceutical were observed in Table 3. From this Table, low standard deviations and good recoveries were observed.

Table 3. Determination of Desogestrel (DSG) and Ethinylestradiol (EE2) in pharmaceutical formulations.

\begin{tabular}{|c|c|c|c|c|c|c|}
\hline \multirow[b]{2}{*}{ Analyte } & \multicolumn{2}{|c|}{ Ciclidon ${ }^{\circledR}$} & \multicolumn{2}{|c|}{ Dal ${ }^{\circledR}$} & \multicolumn{2}{|c|}{ Marvelon ® } \\
\hline & $\begin{array}{l}\text { Nominal mass } \\
(\mathrm{mg})\end{array}$ & $\begin{array}{l}\text { Mass obtained } \\
(\mathrm{mg})^{*}\end{array}$ & $\begin{array}{l}\text { Nominal mass } \\
(\mathrm{mg})\end{array}$ & $\begin{array}{l}\text { Mass obtained } \\
\text { (mg)* }\end{array}$ & $\begin{array}{l}\text { Nominal mass } \\
(\mathrm{mg})\end{array}$ & $\begin{array}{l}\text { Mass obtained } \\
\text { (mg)* }\end{array}$ \\
\hline DSG & 0.150 & $0.148 \pm 3 \cdot 10^{-3}$ & 0.150 & $0.148 \pm 7 \cdot 10^{-4}$ & 0.150 & $0.151 \pm 4 \cdot 10^{-3}$ \\
\hline EE2 & 0.030 & $0.030 \pm 6 \cdot 10^{-4}$ & 0.020 & $0.020 \pm 3 \cdot 10^{-4}$ & 0.030 & $0.032 \pm 7 \cdot 10^{-3}$ \\
\hline Vit.E & --- & --- & --- & --- & --- & 0.09 \\
\hline
\end{tabular}
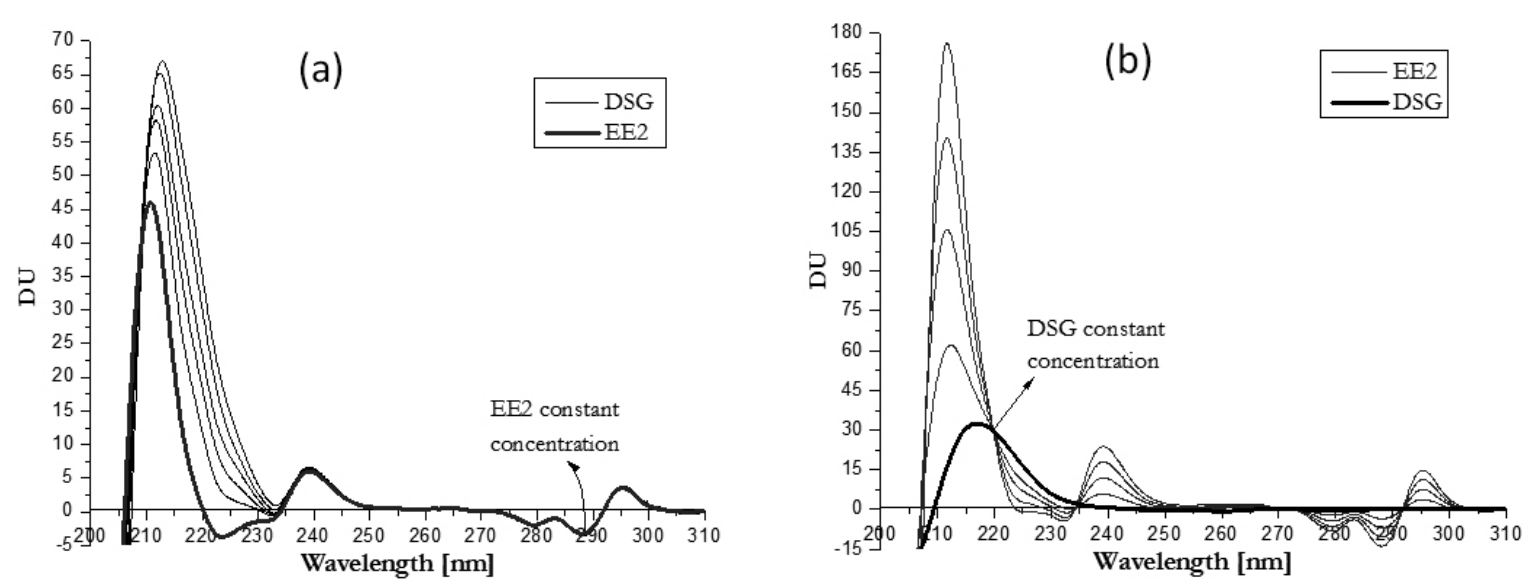

Figure 6. Effect of concentration on the analytical signal for different relationships in mass, (a) concentration range for DSG between $2.0-6.0 \cdot 10^{-5} \mathrm{~mol} / \mathrm{L}$ and EE2 constant concentration of $1.0 \cdot 10^{-5} \mathrm{~mol} / \mathrm{L}$, (b) concentration range for EE2 between $1.0-4.0 \cdot 10^{-5} \mathrm{~mol} / \mathrm{L}$ and DSG constant concentration $5.0 \cdot 10^{-5} \mathrm{~mol} / \mathrm{L}$. 


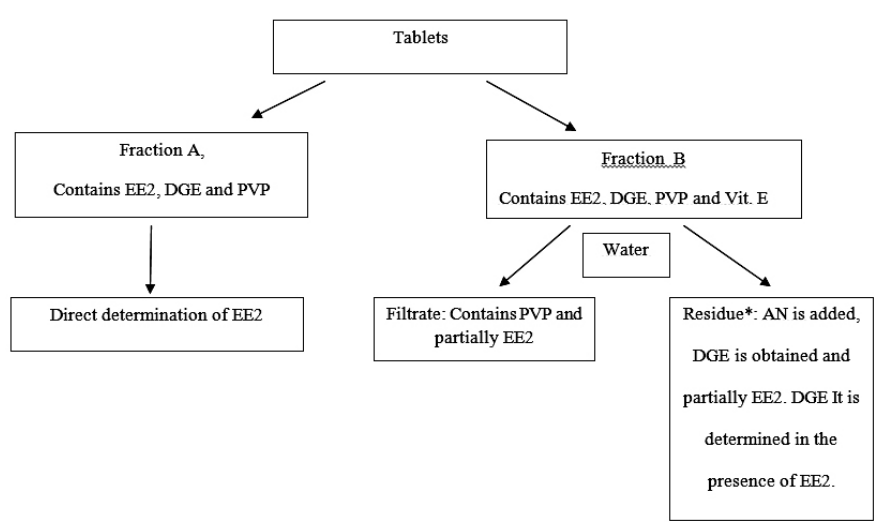

Schema I. Parallel Determination of EE2 y DGE in pharmaceutical formulation.

*If there is present Vit. E, DSG can be determinate by equations system (equation 1) and EE2 at $280.8 \mathrm{~nm}$.

For pharmaceutical formulations containing DSG, EE2, PVP and Vit. E as Neolette $^{\circledR}$ and Marvelon ${ }^{\circledR}$, the developed method can be used for determining DSG in the sample B by resolved a system of equations at 220 and $234 \mathrm{~nm}$ corresponding to the zero-crossing of EE2. It is also possible to determine the concentration of Vit. E in the formulations.

$$
\lambda_{234} \quad D U_{1}=D U_{v i t . E}+D U_{D S G}
$$

Replacing;

$$
\begin{aligned}
& \lambda_{220} \quad D U_{220}=K_{v i t . E}^{220} C_{v i t E}+K_{D S G}^{220} C_{D S G} \\
& \lambda_{234} \quad D U_{234}=K_{v i t . E}^{234} C_{v i t E}+K_{D S G}^{234} C_{D S G}
\end{aligned}
$$

Where:

$D U_{220}=$ Derivative unit value of the tablet at $220 \mathrm{~nm}$

$D U_{234}^{220}=$ Derivative unit value of the tablet at $234 \mathrm{~nm}$.

$K=$ Sensitive value of each compound at different wavelength.

$C=$ Concentration of each analyte.

The results obtained for Marvelon ${ }^{\circledR}$ that contains Vit. E is shown in Table 3. Getting results according to the nominal content in the case of DSG and determine the content of the Vit.E. EE2 can be determined in the fraction A at $280.8 \mathrm{~nm}$ (Figure 4d), according to the following equation 4:

$$
\mathrm{DU}=1,81 * 10^{5} \mathrm{C}+5,8 * 10^{-2}, \mathrm{R}=0,9997
$$

\section{CONCLUSIONS}

Despite that the proposed method is a parallel determination of EE2 and DSG to be applied for quality control in pharmaceutical formulations, this method is rapid, simple, low cost, and possible to implement in a control laboratories.

The developed method for the parallel determination of DSG and EE2 the recovery values obtained were close to $100 \%$, and a RDS close to $2.0 \%$ was observed with a good repeatability, which demonstrates that this method was suitable for the parallel determination of the analytes, eliminating the interference of PVP. Also it is possible to determinate EE2 and DSG in presence of Vit. E in Marvelon ${ }^{\circledR}$, using an equations system for DSG and in a free PVP sample (sample B) at $280.8 \mathrm{~nm}$ for EE2.

\section{ACKNOWLEDGEMENTS}

The authors are grateful to the National Fund for Development of Sciences and Technology (FONDECYT), project 1100103 for financial support and scholarships CONICYT 21120686.

\section{REFERENCES}

1. Z. Yu, B. Xiao, W. Huang, P. Peng, Environ. Toxicol. Chem., 23, 531539, (2004).

2. A. Shareef, M. Angove, J. Wells, B. Johnson, J. Chem. Eng., 51, 879-881, (2006).

3. A.R. Hurwitz, S.T Liu, J. Pharm. Sci., 66, 624-627, (1977).

4. A. Saha, K. Roy, K. De, C. Sengupta, Acta Pol. Pharm., 59, 65-69, (2002).

5. CH. Verhoeven, SF. Krebbers, GN. Wagenaars, RM. Vos, Drug Metab. Dispos., 26, 927-936, (1998).

6. E. Souri, H. Jalalizadeh, H. Farsam, R. Ghadiri, M. Amanlou, Chem. Pharm. Bull., 77, 949-951, (2005).

7. J.J. Berzas, J. Rodríguez, G. Castañeda, F.J. Guzmán, Analyst, 122, 41-44, (1997).

8. J.J.B. Nevado, J.R. Flores, G.C. Penalvo, F.J.G. Bernardo, Anal. Lett, 30, 2221-2233, (1997).

9. J.M. Talmage, MH. Penner, M. Geller, J. Pharm. Sci., 54, 1194-1196, (1965).

10. O.D. Boughton, R. Bryant, W.J. Ludwig, D.L. Timma, J. Pharm. Sci., 55, 951-955, (1966).

11. G. Lunn, HPLC methods for recently approved pharmaceuticals, Vol. 1, Wiley-Vch, Hoboken, New Jersey, 2005; pp. 238-239.

12. M. Inês, R.M. Santoro, N.M. Kassab, M. Hasegawa, E.R. KedorHackmann, Drug Dev. Ind. Pharm., 28, 741-747, (2002).

13. T. Santa, Biomed. Chromatogr., 25, 1-10, (2011)

14. H. Ye, Chinese J. Pharm., 11, 12, (1999).

15. United States Pharmacopeia 33, The United States Pharmacopeial Convention, Rockville, MD (2010)

16. D. Matejícek, V. Kubán, Anal. Chim. Acta., 588, 304-315, (2007).

17. W. Li, Y.H. Li, A.C. Li, S. Zhou, W. Naidong, J. Chromatogr B., 825, 223-232, (2005).

18. T. Korhonen, A. Tolonen, J. Uusitalo, S. Lundgren, J. Jalonen, K. Laine, Br. J. Clin. Pharmacol., 60, 69-75, (2005).

19. R.C. Rowe, P.J. Sheskey, M.E. Quinn, Handbook of Pharmaceutical Excipients, Pharmaceutical Press, London-Chicago, 2009.

20. V. Bühler, Polyvinylpyrrolidone Excipients for Pharmaceuticals: Povidone, Crospovidone and Copovidone, Springer-Verlag, Berlin Heidelberg, New York, 2005.

21. L.A. Turker, F.Y. Guner, O. Guven, Colloid Polym. Sci., 268, 337-344, (1990). 